\title{
Air-suspended $\mathrm{TiO}_{2}$-based $\mathrm{HCG}$ reflectors for visible spectral range
}

\author{
Ehsan Hashemi ${ }^{1}$, Jörgen Bengtsson ${ }^{1}$, Johan Gustavsson ${ }^{1}$, Stefan Carlsson ${ }^{1}$, Georg Rossbach ${ }^{2}$, \\ and Åsa Haglund ${ }^{1}$ \\ ${ }^{1}$ Photonics Laboratory, Department of Microtechnology and Nanoscience, Chalmers University \\ of Technology, Sweden; \\ ${ }^{2}$ Laboratory of Advanced Semiconductors for Photonics and Electronics, Institute of \\ Condensed Matter Physics, École Polytechnique Fédérale de Lausanne (EPFL), Switzerland;
}

\begin{abstract}
For GaN-based microcavity light emitters, such as vertical-cavity surface-emitting lasers (VCSELs) and resonantcavity light emitting diodes (RCLEDs) in the blue-green wavelength regime, achieving a high reflectivity wide bandwidth feedback mirror is truly challenging. The material properties of the III-nitride alloys are hardly compatible with the conventional distributed Bragg reflectors (DBRs) and the newly proposed high-contrast gratings (HCGs). Alternatively, at least for the top outcoupling mirror, dielectric materials offer more suitable material combinations not only for the DBRs but also for the HCGs. HCGs may offer advantages such as transverse mode and polarization control, a broader reflectivity spectrum than epitaxially grown DBRs, and the possibility to set the resonance wavelength after epitaxial growth by the grating parameters. In this work we have realized an air-suspended $\mathrm{TiO}_{2}$ grating with the help of a $\mathrm{SiO}_{2}$ sacrificial layer. The deposition processes for the dielectric layers were fine-tuned to minimize the residual stress. To achieve an accurate control of the grating duty cycle, a newly developed lift-off process, using hydrogen silesquioxan (HSQ) and sacrificial polymethyl-methacrylate (PMMA) resists, was applied to deposit the hard mask, providing sub-10 nm resolution. The finally obtained $\mathrm{TiO}_{2}$ /air HCGs were characterized in a micro-reflectance measurement setup. A peak power reflectivity in excess of $95 \%$ was achieved for TM polarization at the center wavelength of $435 \mathrm{~nm}$, with a reflectivity stopband width of about $80 \mathrm{~nm}$ (FWHM). The measured HCG reflectance spectra were compared to corresponding simulations obtained from rigorous coupled-wave analysis and very good agreement was found.
\end{abstract}

Keywords: vertical-cavity surface-emitting laser, gallium nitride, high-contrast grating, dielectric materials, $\mathrm{TiO} 2$

\section{INTRODUCTION}

A high index-contrast sub-wavelength grating (HCG) is a thin structure with extraordinary features, that is expected to have various implementations in optoelectronic devices. ${ }^{1}$ The most important application of HCGs has been to serve as the top reflector in vertical-cavity surface-emitting lasers (VCSELs), in which they are believed to have several advantages over the already well-established distributed-Bragg reflectors (DBRs). These advantages include interesting properties such as broadband high reflectivity, wavelength setting capability, transverse mode control, and polarization selectivity. Up to now, HCGs have been explored in different material systems and a few electrically pumped VCSELs incorporating an HCG structure have been realized, mostly in GaAs and InP-based material systems emitting at infrared wavelengths. In these experiments, the HCG structures have either been applied as a part of the top reflector, in combination with a couple of DBR pairs, ${ }^{2,3,4}$ or as the one and only part of it, fully substituting the top DBR. ${ }^{5,6}$ One should also mention an optically pumped VCSEL using two HCGs as the top and the bottom reflectors, which proves the concept of DBR-free VCSELs. ${ }^{7}$

In the GaN-based material system, the HCG reflectors are of particular interest since they may potentially solve some of the key challenges regarding VCSELs emitting in the visible. The large lattice-mismatch and/or

(Send correspondence to Ehsan Hashemi)

E-mail: ehsan.hashemi@chalmers.se, Telephone: +46 317721888

High Contrast Metastructures IV, edited by Connie J. Chang-Hasnain, David Fattal,

Fumio Koyama, Weimin Zhou, Proc. of SPIE Vol. 9372, 93720D · C 2015 SPIE

CCC code: $0277-786 \mathrm{X} / 15 / \$ 18 \cdot$ doi: $10.1117 / 12.2078951$

Proc. of SPIE Vol. 9372 93720D-1 
the relative small index-contrast in GaN-based alloys have made it challenging to grow epitaxial DBRs with sufficiently wide bandwidth for the high reflectivity (>99\%) required in VCSELs. In addition to the high theoretical reflectivity of HCGs, post-epitaxial wavelength-setting in the VCSEL can be easily achieved by just changing the grating duty-cycle or period, which can be of interest to partly compensate for growth inhomogeneities or to fabricate VCSELs with individual emission wavelength in one single array.

Unfortunately, the lack of wet-etch selectivity in the GaN material system has made it difficult to directly find a sacrificial material to form an airgap in a membrane type of HCGs. This material constraint has resulted in schemes that do not rely on an airgap, but instead for instance a grating pattern is deeply drilled through the layers of a half-VCSEL structure and transferred into the multi-quantum well layers, ${ }^{8}$ or alternatively a surface grating that has grooves on the surface of the GaN layer. ${ }^{9}$ Although these schemes are mechanically more rigid, the former is not suitable for electrically pumped devices and the latter lacks a high index contrast. To have an airgap underneath the grating layer would increase the width of the reflectivity stopband and simultaneously widen the fabrication tolerance window on the grating parameters. ${ }^{1}$ There have been attempts to realize an airgap, for example by means of a bandgap-selective photoelectrochemical (PEC) etching of InGaN superlattice layers ${ }^{10}$ in which the sacrificial InGaN layers were not sufficiently thick. Alternatively, an airgap underneath the GaN grating structure was fabricated by using a focused-ion-beam (FIB) etching process, ${ }^{11}$ which is inconvenient for large-scale production despite its proof-of-principle approach. Moreover, in the GaN-on-Si platform, ${ }^{12}$ an airgap was obtained by backside $\mathrm{Si}$ wafer etching, ${ }^{13,14}$ by which the integration to VCSELs will remain difficult until the full epitaxial growth of nitrides on $\mathrm{Si}$ with high enough quality is feasible.

In this work, we demonstrate an alternative method to fabricate HCGs suitable for GaN-based blue VCSELs, made of $\mathrm{TiO}_{2}$ grating beams that are freely suspended in air using a $\mathrm{SiO}_{2}$ sacrificial layer. Among dielectric materials, $\mathrm{TiO}_{2}$ is promising thanks to its unique optical, chemical, and mechanical properties. $\mathrm{TiO}_{2}$ has a high refractive index, similar to that of $\mathrm{GaN}$, with a negligible absorption loss at wavelengths longer than $400 \mathrm{~nm}$, its chemical wet-etch selectivity is extremely high in contrast to a $\mathrm{SiO}_{2}$ material, and also the residual mechanical stress in thin films can be tailor-made. Besides, the dielectric HCG scheme employs common material deposition techniques and eliminates the epitaxial growth complications. This $\mathrm{TiO}_{2} /$ air $\mathrm{HCG}$ reflector can be integrated to a selection of different material systems at various emission wavelengths.

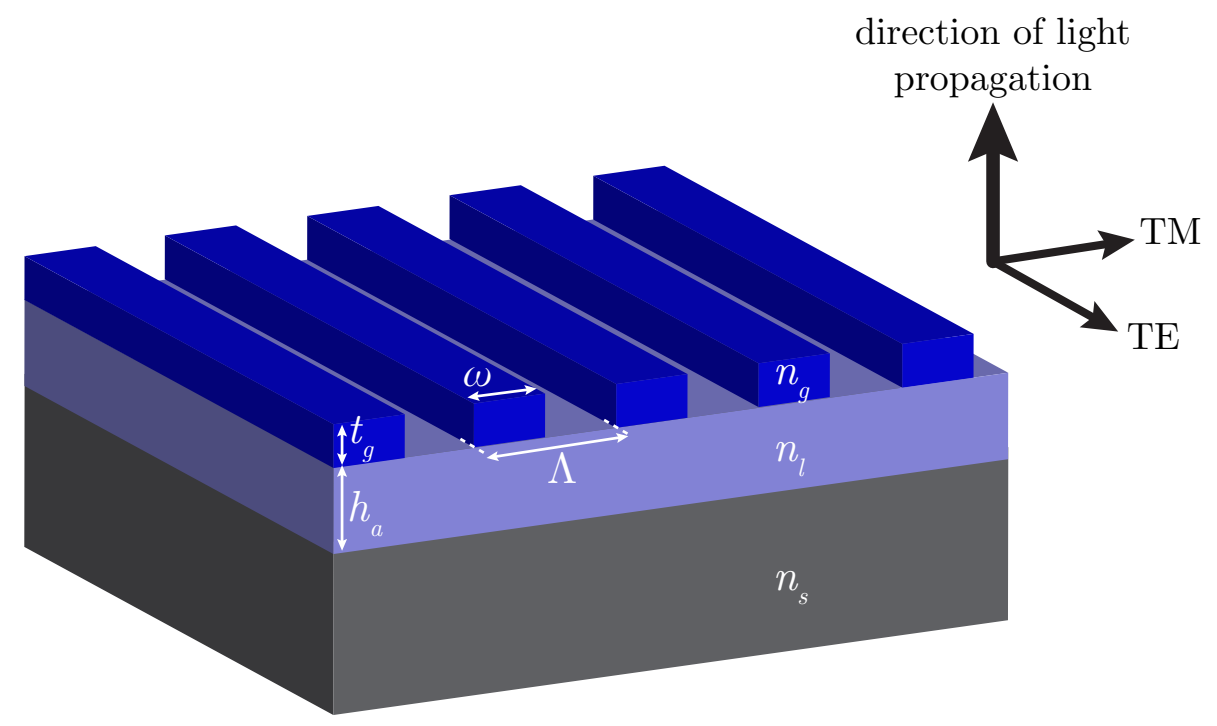

Figure 1: Schematic of HCG with a high index grating material $\left(n_{g}\right)$, low index material $\left(n_{l}\right)$, and substrate index $\left(n_{s}\right)$. HCG parameters are the layer thicknesses $t_{g}$ and $h_{a}$, period $\Lambda$ and duty cycle $\omega / \Lambda$. 


\section{RIGOROUS COUPLED WAVE ANALYSIS}

Numerical simulations have been performed to design the freestanding $\mathrm{TiO}_{2}$ gratings. Rigorous coupled-wave analysis (RCWA) is one of the popular methods for this purpose, which is covered in detail in a work by Moharam, et al. ${ }^{15}$ Among the many implementations of the RCWA method that are freely available, we used the RicWaA MATLAB package. ${ }^{16}$ Figure 1 illustrates the parameters of a typical grating structure model including the wavelength, the polarization of the incoming light, the grating thickness $t_{g}$, the airgap height $h_{a}$, the period $\Lambda$, and the duty-cycle (also called fill-factor) $\omega / \Lambda$. The calculations were limited to a single incident angle that could be varied, although ideally we were interested only in one angle, the normal direction to the interface plane of the $\mathrm{TiO}_{2}$ grating layer and the airgap. An incident plane wave with either a transverse-electric (TE) polarization, along the grating bars, or transverse-magnetic (TM), perpendicular to them, was assumed to impinge on grating beams with identical rectangular cross-sections assuming an infinite number of grating beams which are infinitely long, or at least extending far beyond the area illuminated by the incident light beam.

As a result, the reflectance contour plots (above 99\%) are shown in Fig. 2 for both the TE and TMpolarizations with varying period $\Lambda$ and duty-cycle values. The wavelength was set to $450 \mathrm{~nm}$ at which the refractive index of $\mathrm{TiO}_{2}\left(n_{g}\right)$ is 2.6, taken from ellipsometry measurements, and for the Si substrate the Sellmeier dispersion equation was used. The rest of the parameters have been optimized to yield highest reflectance and widest bandwidth. As can be seen for the TM-polarization, a possible tolerance window for the fabrication imperfection is larger for the TM-polarization design in comparison with the TE-design, which makes the grating performance less sensitive to changes in the $\Lambda$ and duty-cycle.

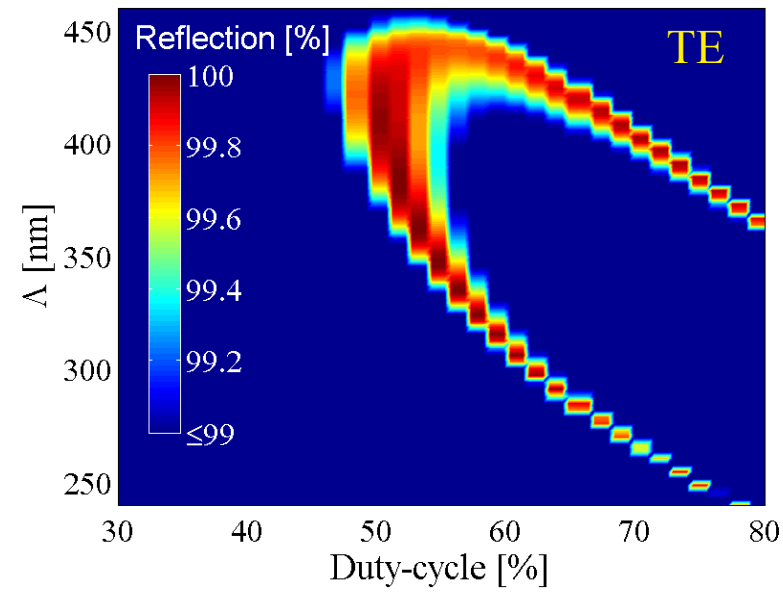

(a)

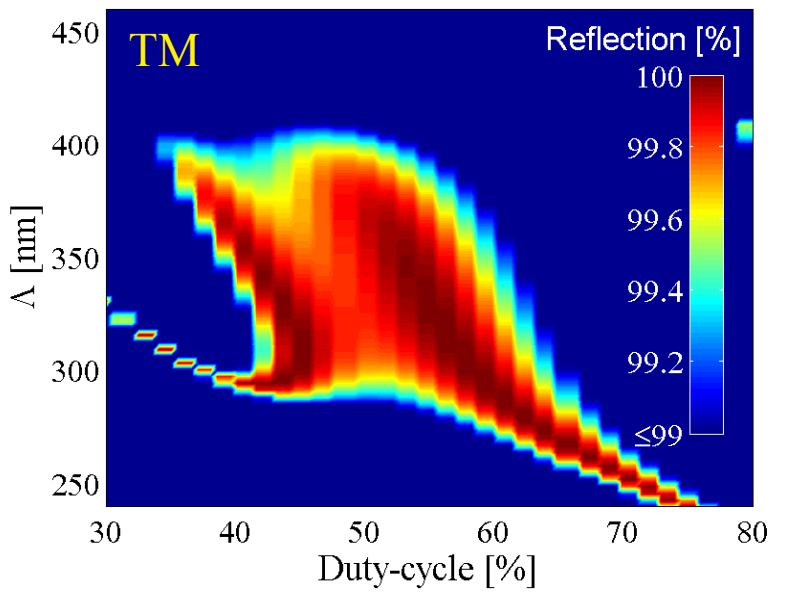

(b)

Figure 2: The reflectance contour plots (above 99\%) for $\mathrm{TiO}_{2}$ /air $\mathrm{HCG}$ using the optimal layer thickness values for (a) TE-mode: $t_{g}=145 \mathrm{~nm}, h_{a}=550 \mathrm{~nm}$, and (b) TM-mode: $t_{g}=205 \mathrm{~nm}, h_{a}=400 \mathrm{~nm}$.

\section{FABRICATION}

Figure 3 schematically summarizes the process steps that enabled the fabrication of the $\mathrm{TiO}_{2}$-based subwavelength free-standing grating structures. Essential to this are the two important considerations that permit successful realization of the structures, namely to control the residual stress in the structure and to use optimized etch recipes.

\subsection{Deposition of dielectric stack}

The first step in the fabrication was the deposition of the dielectric stack. As it can be seen in Fig. 3, in addition to the $\mathrm{SiO}_{2}$ and the $\mathrm{TiO}_{2}$ layers that serve as the sacrificial and the grating layers, an extra $\mathrm{SiO}_{2}$ layer was also used to improve the dry etching profile of the $\mathrm{TiO}_{2}$ gratings and obtain sharp and rectangular-shaped grating beams (cf. Fig. 3(b)). The fabrication details of the dielectric stack are summarized in Table 1. The $\mathrm{SiO}_{2}$ deposited by PECVD was thought to be less dense and thus more suitable for the wet etch removal. Sputtering 


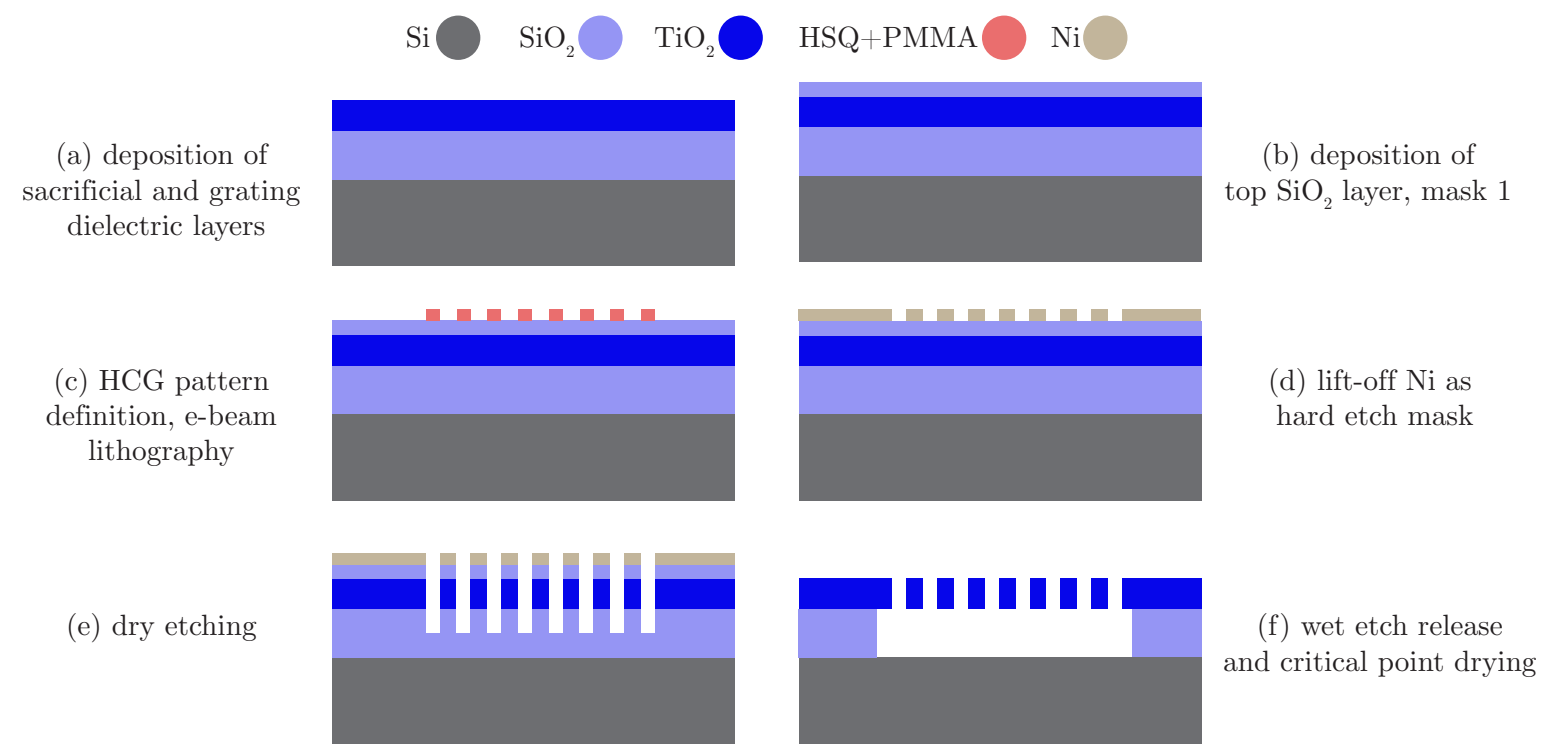

Figure 3: Schematic of the fabrication steps for the $\mathrm{TiO}_{2} /$ air $\mathrm{HCG}$.

was the only method available for the $\mathrm{TiO}_{2}$ deposition, where the $\mathrm{RF}$ bias power applied to the substrate holder could be varied to modify the residual stress of the film. In our initial tests, the as-deposited $\mathrm{TiO}_{2}$ films using the standard deposition parameters $(0 \mathrm{~W}$ RF bias power) resulted in very good optical (high refractive index and low absorption) and chemical (high wet-etch selectivity against $\mathrm{SiO}_{2}$ ) properties, but the mechanical residual stress in the film was too large $(+300 \mathrm{MPa})$. Therefore the released $\mathrm{TiO}_{2}$ grating structures were mostly broken or bent, as can be seen in Fig. 4a,b.

Table 1: Fabrication details of the dielectric stack.

\begin{tabular}{|c|c|c|c|}
\hline $\begin{array}{l}\text { Step } \\
\text { no. }\end{array}$ & Process & Tool & Process parameters \\
\hline 1 & $\begin{array}{l}\text { bowing (stress) measurement on } \\
\text { 2-inch Si substrate }\end{array}$ & $\begin{array}{l}\text { Tencor surface } \\
\text { profilometer }\end{array}$ & - \\
\hline 2 & $400 \mathrm{~nm} \mathrm{SiO}_{2}$ deposition & $\begin{array}{c}\text { PECVD-Oxford } \\
\text { plasmalab } 100 \\
\text { system }\end{array}$ & $\begin{array}{c}8 \mathrm{sccm} \mathrm{SiH}_{4}, 20 \mathrm{sccm} \mathrm{N}_{2} \mathrm{O}, 50 \mathrm{sccm} \mathrm{Ar}, 100 \mathrm{~W} \\
\text { ICP power }\end{array}$ \\
\hline 3 & $\begin{array}{l}\text { bowing (stress) measurement after } \\
\text { deposition }\end{array}$ & $\begin{array}{l}\text { Tencor surface } \\
\text { profilometer }\end{array}$ & - \\
\hline 4 & $220 \mathrm{~nm} \mathrm{TiO}_{2}$ deposition & $\begin{array}{l}\text { Sputtering-FHR } \\
\text { MS150 }\end{array}$ & $\begin{array}{c}1 \mathrm{~kW} \text { dc power, } 5 \cdot 10^{-3} \text { mbar, } 40 \mathrm{sccm} \text { Ar flow, } 4 \\
\text { sccm O2 flow, } 300-400 \mathrm{~W} \text { RF bias power }\end{array}$ \\
\hline 5 & $\begin{array}{l}\text { bowing (stress) measurement after } \\
\text { deposition }\end{array}$ & $\begin{array}{l}\text { Tencor surface } \\
\text { profilometer }\end{array}$ & - \\
\hline 6 & $\mathrm{TiO}_{2}$ film ellipsometry & $\begin{array}{l}\text { J. A. Woollam } \\
\text { M2000 }\end{array}$ & $\begin{array}{l}\text { thickness and refractive index mapping over the } \\
\text { 2-inch wafer }\end{array}$ \\
\hline 7 & $100 \mathrm{~nm} \mathrm{SiO}_{2}$ deposition & $\begin{array}{l}\text { Sputtering-FHR } \\
\text { MS150 }\end{array}$ & $\begin{array}{c}1 \mathrm{~kW} \text { RF power, } 1.3 \cdot 10^{-2} \text { mbar, } 40 \mathrm{sccm} \mathrm{Ar} \\
\text { flow, } 15 \mathrm{sccm} \mathrm{O} 2 \text { flow }\end{array}$ \\
\hline
\end{tabular}

\subsection{Controlling the residual stress}

As mentioned, the residual stress measurement done by simple wafer bow technique revealed $>+300 \mathrm{MPa}$ tensile stress in the initial $\mathrm{TiO}_{2}$ film. However the incorporated stress in the final fabricated gratings after the processing steps should be different, and a more sophisticated technique will be required to determine the stress locally near each structure. ${ }^{17}$ Nevertheless, in our first attempt to cope with the high residual stress in the $\mathrm{TiO}_{2}$ film, a 
stress-relieving trench around the grating was deployed, which in some cases helped to reduce the bending of the grating beams, but unfortunately led to tilting or sagging of the whole structure, see Figs. $4 \mathrm{c}$ and $4 \mathrm{~d}$. Therefore, we had to instead change the deposition conditions of the $\mathrm{TiO}_{2}$ film itself, to reduce the tensile residual stress. Among the tested parameters- the dc power, the RF bias, and the temperature (self-heating)- no dependence of the film stress on the dc power was observed.

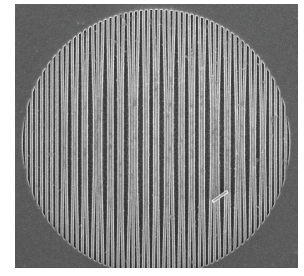

(a)

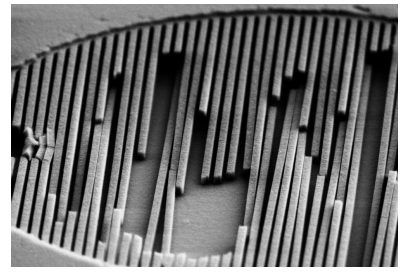

(b)

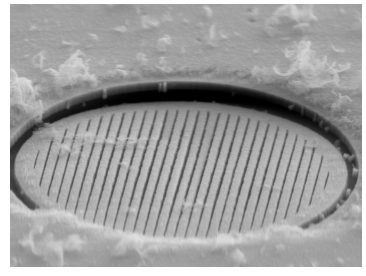

(c)

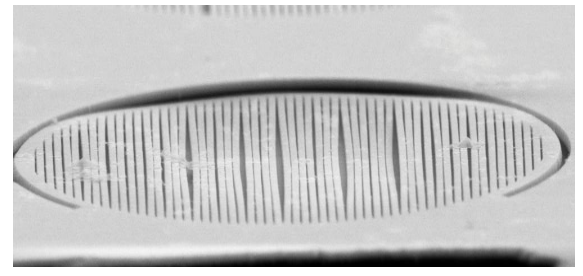

(d)

Figure 4: SEM images of released $\mathrm{TiO}_{2}$ gratings with too large stress in the film $(+300 \mathrm{MPa})$. (a,b) Severe bending of $\mathrm{TiO}_{2}$ grating beams which can even lead to breaking. (c,d) Released $\mathrm{TiO}_{2}$ Gratings with stressrelieving trenches which reduced the bending to some extent, but instead caused tilting and sagging of the whole structure.

We had already observed that thermal annealing could strongly affect the $\mathrm{TiO}_{2}$ film stress and make it more tensile, therefore to produce a low stress film it was important to minimize the self-heating that can occur during the $\mathrm{TiO}_{2}$ sputtering, in which the temperature on the substrate could rise up to $300^{\circ} \mathrm{C}$ in a 30 minute-long deposition run. Therefore, we decided to use several deposition interruptions ( 5 minutes deposition/ 5 minutes cool down in load lock and open air in total) to minimize the heating, and also, as it is illustrated in Fig. 5a, we mounted the wafers with a thermal tape on a copper chuck to improve the heat dissipation. The mounting could also result in a more uniform temperature distribution across the wafer and an improved stress uniformity.

Besides the effect of self heating, an RF bias power supplied to the substrate holder could strongly change the conditions of the film deposition, where a higher RF bias power resulted in more compressively stressed films. A number of tests were carried out with different RF bias values, and the resulting film stress in the wafers was measured using a wafer bowing technique, and the results are plotted in Fig. 5b. The optimum RF bias was found to be around $300 \mathrm{~W}$ which resulted in a slightly compressive stress in the film. No bending or buckling could be observed in the finalized released gratings whose $\mathrm{TiO}_{2}$ films had an initial compressive stress ranging from 0 to $-100 \mathrm{MPa}$.

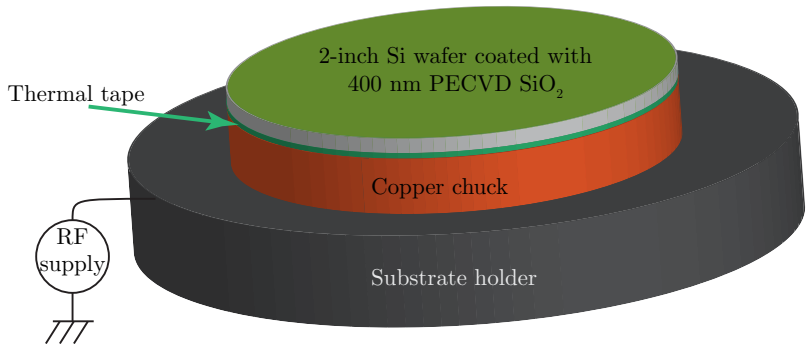

(a)

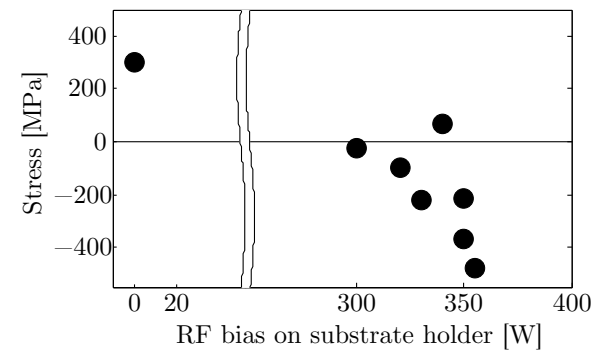

(b)

Figure 5: (a) The wafers were mounted with thermal tape on a copper chuck to improve the heat dissipation and minimize the effect of deposition self-heating on the tensile stress build-up in the $\mathrm{TiO}_{2}$ film. (b) Stress in the $\mathrm{TiO}_{2}$ film as a function of different RF power supplied to the substrate.

\subsection{Pattern definition and Ni lift-off}

The patterns that are designed for the HCGs were defined on the sample using an Jeol JBX 9300 electron beam (e-beam) lithography system. A bilayer negative e-beam resist made of the combination of hydrogen silesquioxan 
(HSQ) and poly-methyl-methacrylate (PMMA-A2) was utilized as it has proven well suited for the subsequent Ni lift-off process. ${ }^{18} 50 \mathrm{~nm}$ thick $\mathrm{Ni}$ was evaporated onto the developed resist using an e-beam evaporation system followed by lift-off to create the required hard mask for the etching process.

\subsection{Dry etching and wet etch sacrificial removal}

Metal-oxides including $\mathrm{TiO}_{2}$ and $\mathrm{SiO}_{2}$ are typically etched with fluorine based plasmas, among which $\mathrm{CHF}_{3}$ has the advantage of forming controlled amount of fluoro-carbon polymers that can help protecting the side walls. ${ }^{19,20}$ The grating patterns were etched into the $\mathrm{TiO}_{2}$ layer in an inductively coupled plasma (ICP)/ reactive ion etch (RIE) system (Oxford Plasmalab 100). A laser interferometer for in-situ monitoring was employed to stop the etch in the underlying $\mathrm{SiO}_{2}$ sacrificial layer. A low pressure of $<5$ mTorr, $75 \mathrm{~W}$ of electrode RF power, and ICP power of $125 \mathrm{~W}$ led to very good etch rate control and directionality in a way that sharp edges of $\mathrm{TiO}_{2}$ grating were obtained.

After etching the cross-sections of the gratings were characterized using Scanning Electron Microscopy (SEM) to control not only the side walls of the grating but also to ensure that the etch had been continued deep into the bottom $\mathrm{SiO}_{2}$ layer. A too shallow etch into the bottom $\mathrm{SiO}_{2}$ layer would make the following wet etch removal of the sacrificial layer more difficult and time consuming.

The Ni hard mask was first removed using a $\mathrm{Cr} / \mathrm{Ni}$ etchant. In order to ensure that the $\mathrm{Ni}$ is entirely removed, the samples were submerged in a $60^{\circ} \mathrm{C} \mathrm{Cr} / \mathrm{Ni}$ solution for about 15 minutes. After that the wet etch removal of the sacrificial $\mathrm{SiO}_{2}$ layer was immediately performed utilizing a diluted mixture of buffered-oxide-etch (BOE) and deionized water with a 1:5 ratio for 4 minutes to selectively etch away the $\mathrm{SiO}_{2}$ and release the grating structures. Finally the samples had to undergo a $\mathrm{CO}_{2}$ critical point drying step to avoid collapse of the grating beams due to bonding to the substrate by the capillary forces.

The final freestanding $\mathrm{TiO}_{2} \mathrm{HCG}$ with a period of $350 \mathrm{~nm}$ and duty cycle of about $50 \%$ is shown in Fig. 6 .

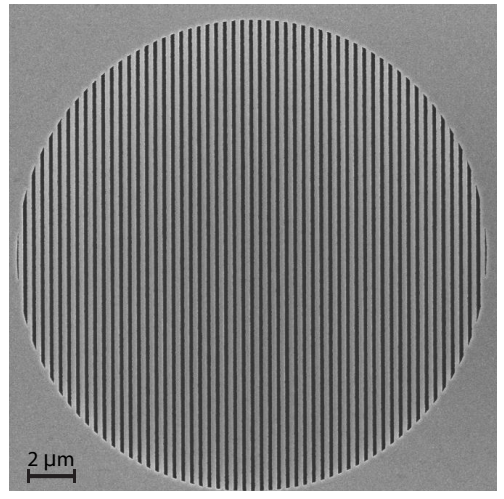

(a)

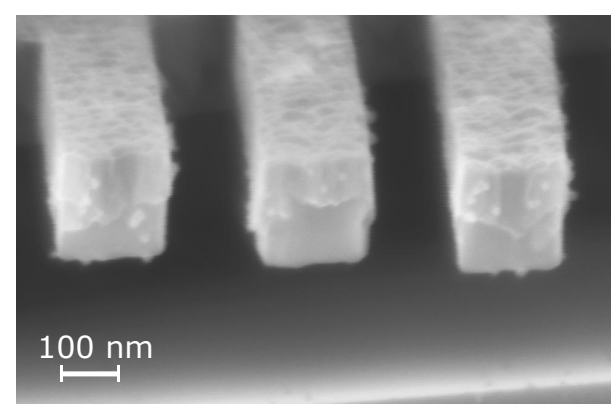

(b)

Figure 6: SEM images of the final fabricated freestanding $\mathrm{TiO}_{2} \mathrm{HCG}$ with a period of $350 \mathrm{~nm}$ and duty-cycle of about $50 \%$ in (a) top view and (b) cross-sectional view.

\section{OPTICAL CHARACTERIZATION}

The fabricated HCGs are small, their diameters $<20 \mu \mathrm{m}$, and designed for normal incident reflection. This makes the characterization and reflectivity measurement of the HCGs a very challenging task. The incoming light from a white light source must have a spot size less than the diameter of the HCG, and impinge on the grating surface with a normal angle. Therefore, a microscope objective with a $100 \times$ magnification was used to focus the beam down to a $10 \mu \mathrm{m}$ diameter. In order to achieve almost normal incidence and good comparability with the simulation, the initial numerical aperture $(\mathrm{NA}=0.5)$ of the objective was artificially reduced to an acceptance angle of $-6^{\circ}<\theta<+6^{\circ}$ by a $1.5 \mathrm{~mm}$ pinhole placed in front of the objective opening. Moreover a polarizer was inserted in the light path to allow for characterization of the polarization selectivity of the HCG, see Fig. 7a. 
Figure $7 \mathrm{~b}$ shows the measured and simulated reflectance spectra of the $\mathrm{TiO}_{2}$ /air $\mathrm{HCG}$ on a Si substrate for both TM- and TE-modes. Reflectance values in excess of $95 \%$ with a full-width at half-maximum (FWHM) stopband of over $80 \mathrm{~nm}$ are obtained for the TM-mode at the center wavelength of $435 \mathrm{~nm}$. For the TE-mode, however, the peak reflectance is almost $30 \%$ lower. Furthermore, the RCWA simulations can very well reproduce the measured spectra using the actual fabricated dimensions measured by SEM, the refractive index dispersion of $\mathrm{TiO}_{2}$ from previously obtained ellipsometry data, and by including a Si substrate in the structure. The finite acceptance angle is accounted for by averaging the simulated reflectance spectra for all different angles within the range of the acceptance angle.

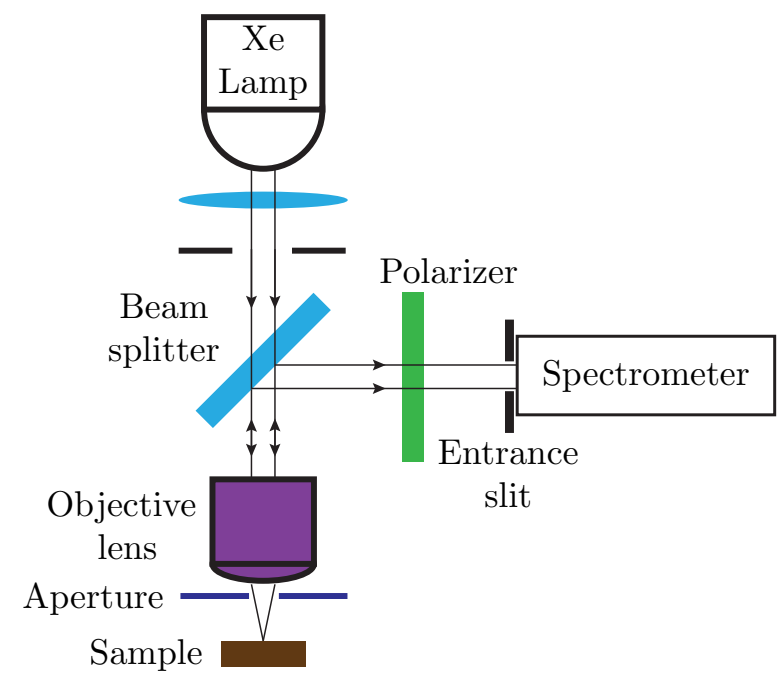

(a)

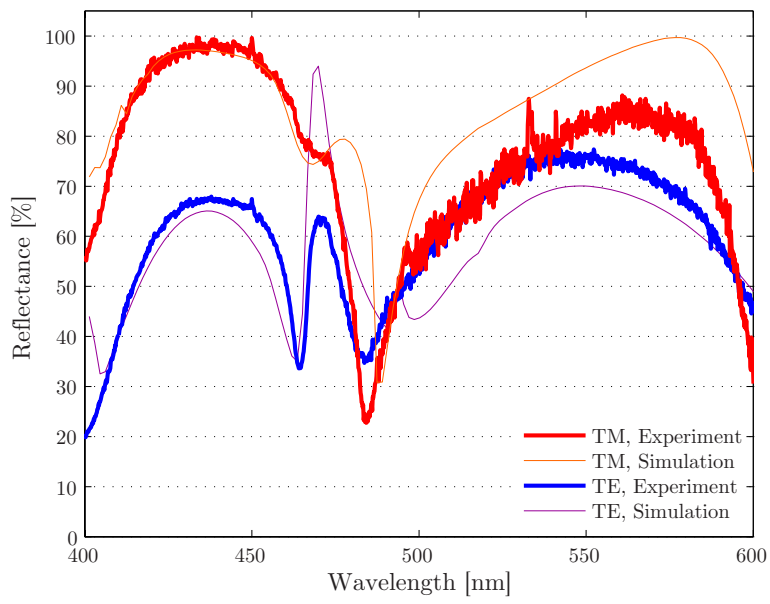

(b)

Figure 7: (a) Schematic illustration of the micro-reflectance setup. (b) Measured and simulated reflectance spectra for both TM and TE modes of the $\mathrm{TiO}_{2}$ HCGs with a period of $370 \mathrm{~nm}$ and duty cycle of about $45 \%$.

\section{SUMMARY}

In summary we have demonstrated that $\mathrm{TiO}_{2}$ has the optical, chemical, and mechanical properties that make it a very suitable material for air-suspended HCGs. The deposition of the $\mathrm{TiO}_{2}$ film and the etching were developed to yield gratings without twisting or bending of the beams, and with near-ideal rectangular beam cross sections. Optical evaluation showed very good agreement with simulations, with a high peak reflectivity ( $>95 \%)$ and a large reflectivity bandwidth $(80 \mathrm{~nm}$ FWHM) for the TM polarization. We believe that this type of reflector can simplify the realization of feedback elements in GaN-based VCSELs and resonant LEDs emitting at visible wavelengths.

\section{ACKNOWLEDGMENTS}

The authors would like to thank Henrik Frederiksen, Mats Hagberg, and Bengt Nilsson from the MC2 Nanofabrication Laboratory at Chalmers University of Technology for technological support in processing, and Nicolas Grandjean at EPFL in Switzerland for providing the micro-reflectance measurement setup. This work was funded by the Swedish Research Council and the Hasselblad Foundation.

\section{REFERENCES}

1. C. J. Chang-Hasnain and W. Yang, "High-contrast gratings for integrated optoelectronics," Advances in Optics and Photonics 4(3), pp. 379-440, 2012.

2. M. C. Huang, Y. Zhou, and C. J. Chang-Hasnain, "A surface-emitting laser incorporating a high-indexcontrast subwavelength grating," Nature Photonics 1(2), pp. 119-122, 2007. 
3. P. Gilet, N. Olivier, P. Grosse, K. Gilbert, A. Chelnokov, I.-S. Chung, and J. Mørk, "High-index-contrast subwavelength grating VCSEL," Proc. SPIE 7615, pp. 76150J-76150J-8, 2010.

4. C. Chase, Y. Rao, W. Hofmann, and C. J. Chang-Hasnain, "1550 nm high contrast grating VCSEL," Optics Express 18(15), pp. 15461-15466, 2010.

5. T. Ansbæk, I.-S. Chung, E. S. Semenova, and K. Yvind, "1060-nm Tunable Monolithic High Index Contrast Subwavelength Grating VCSEL," Photonics Technology Letters, IEEE 25(4), pp. 365-367, 2013.

6. W. Hofmann, C. Chase, M. Muller, Y. Rao, C. Grasse, G. Bohm, M.-C. Amann, and C. J. ChangHasnain, "Long-wavelength high-contrast grating vertical-cavity surface-emitting laser," Photonics Journal, IEEE 2(3), pp. 415-422, 2010.

7. C. Sciancalepore, B. B. Bakir, S. Menezo, X. Letartre, D. Bordel, and P. Viktorovitch, "III-V-on-Si Photonic Crystal Vertical-Cavity Surface-Emitting Laser Arrays for Wavelength Division Multiplexing," Photonics Technology Letters, IEEE 25(12), pp. 1111-1113, 2013.

8. T. T. Wu, S. H. Wu, T. C. Lu, and S. C. Wang, "GaN-based high contrast grating surface-emitting lasers," Applied Physics Letters 102(8), p. 081111, 2013.

9. J. Lee, S. Ahn, H. Chang, J. Kim, Y. Park, and H. Jeon, "Polarization-dependent GaN surface grating reflector for short wavelength applications," Optics Express 17(25), pp. 22535-22542, 2009.

10. J. Kim, D.-U. Kim, J. Lee, H. Jeon, Y. Park, and Y.-S. Choi, "AlGaN membrane grating reflector," Applied Physics Letters 95(2), p. 021102, 2009.

11. T. T. Wu, Y. C. Syu, S. H. Wu, W. T. Chen, T. C. Lu, S. C. Wang, H. P. Chiang, and D. P. Tsai, "Subwavelength GaN-based membrane high contrast grating reflectors," Optics Express 20(18), pp. 20551-20557, 2012.

12. N. V. Triviño, G. Rossbach, U. Dharanipathy, J. Levrat, A. Castiglia, J.-F. Carlin, K. Atlasov, R. Butté, R. Houdré, and N. Grandjean, "High quality factor two dimensional GaN photonic crystal cavity membranes grown on silicon substrate," Applied Physics Letters 100(7), p. 071103, 2012.

13. Y. Wang, T. Wu, T. Tanae, H. Zhu, and K. Hane, "The resonant III-nitride grating reflector," Journal of Micromechanics and Microengineering 21(10), p. 105025, 2011.

14. Y. Wang, Z. Shi, X. Li, S. He, M. Zhang, and H. Zhu, "Surface-normal emission from subwavelength GaN membrane grating," Optics express 22(1), pp. 667-672, 2014.

15. M. G. Moharam, T. K. Gaylord, E. B. Grann, and D. A. Pommet, "Formulation for stable and efficient implementation of the rigorous coupled-wave analysis of binary gratings," J. Opt. Soc. Am. A 12, pp. 10681076, May 1995.

16. L. Zhang, "RicWaA 1.0.2 MATLAB package," 2010 (accessed 24/9/2013). http://www-personal.umich . edu/ zlei/.

17. J. Laconte, D. Flandre, and J. P. Raskin, "Thin dielectric films stress extraction," Micromachined Thin-Film Sensors for SOI-CMOS Co-Integration, pp. 47-103, 2006.

18. M. Rommel, B. Nilsson, P. Jedrasik, V. Bonanni, A. Dmitriev, and J. Weis, "Sub-10nm resolution after lift-off using HSQ/PMMA double layer resist," Microelectronic Engineering 110, pp. 123-125, 2013.

19. A. N. Noemaun, F. W. Mont, J. Cho, E. F. Schubert, G. B. Kim, and C. Sone, "Inductively coupled plasma etching of graded-refractive-index layers of $\mathrm{TiO}_{2}$ and $\mathrm{SiO}_{2}$ using an ITO hard mask," Journal of Vacuum Science $\&$ Technology A 29(5), p. 051302, 2011.

20. N. Rueger, J. Beulens, M. Schaepkens, M. Doemling, J. Mirza, T. Standaert, and G. Oehrlein, "Role of steady state fluorocarbon films in the etching of silicon dioxide using $\mathrm{CHF}_{3}$ in an inductively coupled plasma reactor," Journal of Vacuum Science \& Technology A 15(4), pp. 1881-1889, 1997. 